\title{
The influence of ultra-fast laser pulses on electron transfer in molecular wires studied by a non-Markovian density matrix approach
}

\author{
Sven Welack $*$ Michael Schreiber, and Ulrich Kleinekathöfer \\ Institut für Physik, Technische Universität Chemnitz, 09107 Chemnitz, Germany
}

(Dated: October 8, 2018)

\begin{abstract}
New features of molecular wires can be observed when they are irradiated by laser fields. These effects can be achieved by periodically oscillating fields but also by short laser pulses. The theoretical foundation used for these investigations is a density matrix formalism where the full system is partitioned into a relevant part and a thermal fermionic bath. The derivation of a quantum master equation, either based on a time-convolutionless or time-convolution projection-operator approach, incorporates the interaction with time-dependent laser fields non-perturbatively and is valid at low temperatures for weak system-bath coupling. From the population dynamics the electrical current through the molecular wire is determined. This theory including further extensions is used for the determination of electron transport through molecular wires. As examples, we show computations of coherent destruction of tunneling in asymmetric periodically driven quantum systems, alternating currents and the suppression of the directed current by using a short laser pulse.
\end{abstract}

\section{INTRODUCTION}

Intra- and intermolecular electron transfer has been studied for several decades 1.2 .3 including its coherent control ${ }^{4}$. Lately the closely related field of molecular electronics has attracted much interest, especially the transport through molecular wires5.6. Experimental progress ${ }^{7.8 .9}$ made in this field also spurred a large theoretical effort ${ }^{5.6}$. Many of those theoretical studies are based on a tight-binding model for the wire. The current-voltage characteristics is calculated either using a scattering approach ${ }^{10}$ or an electron transfer scheme ${ }^{11}$ while both formalisms have the same roots 12 . The wellknown Landauer-Büttiker scattering formalism ${ }^{13.14}$ provides a method to compute the steady-state currents in systems on the nanoscale connecting two or more electrodes and later extensions of the formalism also deal with oscillating fields ${ }^{15.16}$. Many theories are based on the assumption of a weak coupling of the wire to the leads and employ a perturbation theory in this small parameter $17.18,19.20$. In this aspect these approaches are very similar to Redfield-like approaches in the field of dissipative quantum dynamics. Furthermore, in some theories the wire is coupled to a dissipative environment to mimic relaxation and decoherence in the wire $21,22,23.24$ or to determine current-induced light emission ${ }^{25}$. Additional effects are observed when the molecular wire is irradiated by a periodic laser field ${ }^{17,24,26,27}$. Only very little is known for non-periodic driving fields 18,28 . One of these studies 28 uses an extension of density functional theory to describe time-dependent transport phenomena.

In this contribution we are also interested in a description of the current through a molecular wire which is based on a quantum master equation. The aim is to extend those methods and results obtained for periodic

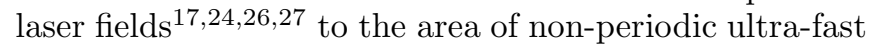

*Electronic address: sven.welack@s2000.tu-chemnitz.de laser pulses. To do so we borrow techniques developed in the area of dissipative quantum dynamics. Instead of coupling the system, i.e. the wire, to a bosonic heat bath it is coupled to a fermionic particle reservoir with which it can exchange particles. Externally applied optical fields can influence the dynamics in a direct manner by changing the wire part but also in an indirect way by influencing the wire-lead coupling. In the area of dissipative quantum dynamics these processes have been mainly studied for monochromatic laser fields $29,30,31.32 .33$ but also for short laser pulses ${ }^{34.35}$. While for monochromatic fields one often employs Floquet states, as for the transport in periodically driven wires $17,24,26,27$, the situation is more complex for non-periodic laser pulses. Meier and Tannor ${ }^{35}$ proposed a method how to use a special parametrization of the so-called spectral density of the reservoir leading to a set of coupled equations for a primary and several auxiliary density matrices. Within this approach one automatically accounts for the influence of the external field on the dissipation operator. The technique of Meier and Tannor is based on the time-nonlocal (TNL) Nakajima-Zwanzig identity ${ }^{36.37}$, while a similar scheme with auxiliary operators has been developed also for time-local (TL) quantum master equations ${ }^{38.39}$. A similar technique, i.e. a direct decomposition of the correlation function, was introduecd by Korolkov and Paramanov in earlier work ${ }^{40}$.

In the current work the technique of auxiliary density matrices is used in the framework of molecular wires. The model Hamiltonian employed for this purpose is introduced in the following section. Section III shows the evolution of the density matrix using a decomposition of the spectral density while Section IV is dedicated to the derivation of the current equation used for the numerical calculations. In Section V, we will make a few comments on TNL versus TL theory. The formalism is tested for periodic and non-periodic laser fields in section VI. The last section gives a summary and outlook. 


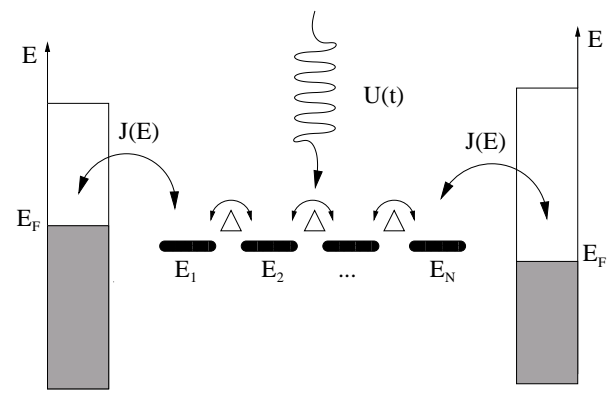

FIG. 1: A multi-site system is studied in which the two outermost sites are attached to electronic reservoirs in thermal equilibrium with their respective Fermi energies $E_{F, l}$ and $E_{F, r}$. The coupling of the outermost sites to the corresponding lead is described by a spectral density function $J_{R}(\omega)$ and the different sites are connected to each other by a hopping element $\Delta$. The on-site energies $E_{i}$ of the wire can be manipulated with a time-dependent external electric field $U(t)$.

\section{MODEL}

The system of interest can be represented in a very general form by a time-dependent Hamiltonian

$$
H(t)=H_{S}(t)+H_{R}+H_{S R},
$$

which consists of the time-dependent part describing the relevant system $H_{S}(t)$, a Hamiltonian describing the environment $H_{R}$, in the presented study the electronic leads, and a coupling term $H_{S R}$ between the relevant system and its environment. The wire is formed by electronic sites $n$ coupled to each other by a hopping element $\Delta$. In second quantization, this orbital tight-binding description of the molecular wire reads

$$
H_{S}(t)=\sum_{n n^{\prime}} H_{n n^{\prime}}(t) c_{n}^{\dagger} c_{n^{\prime}}
$$

where $c_{n}$ annihilates and $c_{n}^{\dagger}$ creates an electron at site $n$ with the anticommutator $\left[c_{n}^{\dagger}, c_{n^{\prime}}\right]_{+}=\delta_{n, n^{\prime}}$. Here, the time-dependence of $H_{S}(t)$ occurs only in to the irradiation of the system by an external electromagnetic field that manipulates the on-site energies $E_{n}$ with a timedependent on-site potential $U_{n}(t)$. By neglecting possible influences of the external field on the tight-binding hopping element $\Delta$, the tight-binding matrix elements $H_{n n^{\prime}}(t)$ can be decomposed into

$$
H_{n n^{\prime}}(t)=-\Delta\left(\delta_{n+1, n^{\prime}}+\delta_{n, n^{\prime}+1}\right)+\left(E_{n}+U_{n}(t)\right) \delta_{n n^{\prime}} .
$$

The environment of the wire consists of two electronic leads that are modeled by two independent electron reservoirs in thermal equilibrium. For each lead, the Hamiltonian $H_{R}$ in second quantization is given by

$$
H_{R}=\sum_{q} \omega_{q} c_{q}^{\dagger} c_{q}
$$

with $c_{q}^{\dagger}$ and $c_{q}$ creating and annihilating an electron in the corresponding reservoir mode $|q\rangle$ with mode energy $\omega_{q}$. Due to the assumed thermal equilibrium of the electronic leads, the occupation expectation values of the reservoir modes are determined by

$$
\left\langle c_{q}^{\dagger} c_{q^{\prime}}\right\rangle=n_{F}\left(\omega_{q}-E_{F}\right) \delta_{q q^{\prime}},
$$

where $n_{F}$ is the Fermi function and $E_{F}$ the Fermi energy. The left electronic lead is coupled to the first site and the right one to the last site $N$ of the wire. To keep the notation simple, we will only refer to the left lead in further derivations but the formalism has to be applied to the right lead as well. The coupling of the left electronic lead with the first site of the wire reads in second quantization

$$
H_{S R}=\sum_{q}\left(V_{q} c_{1}^{\dagger} c_{q}+V_{q}^{*} c_{q}^{\dagger} c_{1}\right)
$$

with a system-lead coupling strength $V_{q}$ for each reservoir mode. In general, these coupling values are determined by the electronic bands of the leads, e.g. the gold contacts, that couple with the energy levels of the wire and by the occupation level of these bands with electrons given by the Fermi energy and the corresponding Fermi function.

\section{REDUCED DENSITY MATRIX AND TIME-NONLOCAL APPROACH}

\section{A. Equation of motion}

In general, the equation of motion (EOM) for the complete density operator $\rho(t)$ including the wire and the leads is given by the Liouville equation

$$
\frac{\partial \rho(t)}{\partial t}=-\frac{i}{\hbar}[H(t), \rho(t)]=-\frac{i}{\hbar} \mathcal{L}(t) \rho(t) .
$$

But the information of interest is limited only to the system part of the density operator $\rho_{S}(t)$, i.e. the wire part, which can be obtained by defining a projection operator $P$ projecting the complete system onto the relevant part and by tracing out the reservoir degrees of free$\operatorname{dom} P \rho(t)=\rho_{R} \operatorname{tr}_{R}\{\rho(t)\}=\rho_{R} \otimes \rho_{S}(t)$. The irrelevant part can be determined by the projection $Q=1-P$. Solving the EOM for the irrelevant part to express the EOM of the relevant part leads directly to the NakajimaZwanzig 36.37 .41 operator identity

$$
\begin{aligned}
P \dot{\rho}= & -i P \mathcal{L}(t) P \rho(t) \\
& -P \mathcal{L}(t) \int_{t_{0}}^{t} \mathrm{~d} t^{\prime} \vec{T} e^{-i \int_{t^{\prime}}^{t} \mathrm{~d} \tau Q \mathcal{L}(\tau)} Q \mathcal{L}\left(t^{\prime}\right) P \rho\left(t^{\prime}\right),
\end{aligned}
$$

where the initial-value term is neglected. Since all the operators are chronologically ordered in time, the literature refers to this TNL approach often as chronological time ordering prescription (COP $)^{42.43,44}$ or time convolution approach ${ }^{45}$. In order to derive an applicable 
method to solve Eq. (8), we apply second order perturbation theory ${ }^{35.46 .47}$ and trace over the reservoir degrees of freedom to get

$$
\begin{aligned}
\dot{\rho}_{S}(t)= & -i \mathcal{L}_{S}(t) \rho_{S}(t) \\
& -\operatorname{tr}_{R}\left\{\mathcal{L}_{S R}(t) \int_{t_{0}}^{t} \mathrm{~d} t^{\prime} U_{0}\left(t, t^{\prime}\right) \mathcal{L}_{S R}\left(t^{\prime}\right) \rho\left(t^{\prime}\right)\right\} .
\end{aligned}
$$

Here $\mathcal{L}_{S}, \mathcal{L}_{R}$ and $\mathcal{L}_{S R}$ are the Liouville operators which, respectively, apply $H_{S}, H_{R}$ and $H_{S R} . \quad U_{0}\left(t, t^{\prime}\right)=$ $\vec{T} e^{-\frac{i}{\hbar} \int_{t^{\prime}}^{t} \mathrm{~d} \tau\left[\mathcal{L}_{S}(\tau)+\mathcal{L}_{R}\right]}$ is the time evolution operator of the full system without the interaction part $H_{S R}$ and $\vec{T}$ is the time ordering operator in positive time direction. The annihilation and creation operators of the system and the environment are defined in different Hilbert spaces which makes it possible to rewrite the coupling Hamiltonian in Eq. (6) as

$$
H_{S R}=\sum_{x=1}^{2} K_{x} \otimes \Phi_{x}
$$

with $\Phi_{1}=\sum_{q} V_{q} c_{q}, \Phi_{2}=\sum_{q} V_{q}^{*} c_{q}^{\dagger}$ and $K_{1}=c_{1}^{\dagger}$, $K_{2}=c_{1}$. Inserting Eq. (10) in Eq. (9) and using the definition of the Liouville operators, a non-Markovian quantum master equation in the Schrödinger picture is obtained $19,20,39,48$

$$
\begin{aligned}
\dot{\rho}_{S}(t)= & -i \mathcal{L}_{S}(t) \rho_{S}(t) \\
& -\int_{t_{0}}^{t} \mathrm{~d} t^{\prime} \sum_{x x^{\prime}} \operatorname{tr}_{R}\left\{\left[K_{x} \Phi_{x}, U_{s}\left(t, t^{\prime}\right) e^{-i H_{R}\left(t-t^{\prime}\right)}\right.\right. \\
& \left.\left.\times\left[K_{x^{\prime}} \Phi_{x^{\prime}}, \rho_{S}\left(t^{\prime}, t_{0}\right)\right] e^{i H_{R}\left(t-t^{\prime}\right)}\right]\right\}
\end{aligned}
$$

with $U_{S}\left(t, t^{\prime}\right)=T_{+} e^{-\frac{i}{\hbar} \int_{t^{\prime}}^{t} \mathrm{~d} \tau \mathcal{L}_{S}(\tau)}$ being the time evolution operator of the relevant system.

The trace over the lead degrees of freedom and all the reservoir operators in the dissipation term can be summarized into correlation functions

$$
C_{x x^{\prime}}(t)=\operatorname{tr}_{R}\left\{e^{i H_{R} t} \Phi_{x} e^{-i H_{R} t} \Phi_{x^{\prime}} \rho_{R}\right\}
$$

that contain all the information about the reservoir and its interaction with the corresponding wire site. For the system of interest, these correlation functions decay in time thereby causing a memory loss in the dissipation term of Eq. (11).

Due to the thermal equilibrium condition of the electronic leads, two of the four functions are zero, $C_{11}=$ $C_{22}=0$. This reduces the summation over $x$ and $x^{\prime}$ to the pairs $\left(x x^{\prime}\right)=(12)$ and $\left(x x^{\prime}\right)=(21)$. Using the property of the correlation functions that $C_{x x^{\prime}}(t)=C_{x x^{\prime}}^{*}(-t)$, we can define the following auxiliary operators

$$
\begin{aligned}
& \Lambda_{x x^{\prime}}(t)=\int_{t_{0}}^{t} \mathrm{~d} t^{\prime} C_{x x^{\prime}}\left(t-t^{\prime}\right) U_{S}\left(t, t^{\prime}\right) K_{x^{\prime}} \rho_{S}\left(t^{\prime}\right), \\
& \widehat{\Lambda}_{x x^{\prime}}(t)=\int_{t_{0}}^{t} \mathrm{~d} t^{\prime} C_{x^{\prime} x}^{*}\left(t-t^{\prime}\right) U_{S}\left(t, t^{\prime}\right) \rho_{S}\left(t^{\prime}\right) K_{x^{\prime}}
\end{aligned}
$$

to simplify Eq. (11). These auxiliary operators incorporate the memory of the system and weight the timedependent electron transfer between the wire and the lead. Expressing the quantum master equation (11) in terms of the auxiliary operators, we get the final expression for the master equation of the reduced density operator at time $t$

$$
\dot{\rho}_{S}(t)=-i \mathcal{L}_{S}(t) \rho_{S}(t)-\sum_{x x^{\prime}}\left[K_{x}, \Lambda_{x x^{\prime}}(t)-\widehat{\Lambda}_{x x^{\prime}}(t)\right] .
$$

\section{B. Spectral decomposition}

In analogy to methods recently developed for systems coupled to a bosonic bath ${ }^{19.20 .35 .39}$, we develop equations of motions for the auxiliary operators defined by Eqs. [13] 14) using a numerical decomposition of the spectral density $J_{R}(\omega)$ to decompose the reservoir correlation functions. Solving the trace in Eq. (12) and making use of Eq. (5), the nonvanishing correlation functions read

$$
\begin{gathered}
C_{12}(t)=\sum_{q}\left|V_{q}\right|^{2} n_{F}\left(-\omega_{q}+E_{F}\right) e^{-i \omega_{q} t} \\
C_{21}(t)=\sum_{q}\left|V_{q}\right|^{2} n_{F}\left(\omega_{q}-E_{F}\right) e^{i \omega_{q} t} .
\end{gathered}
$$

All the external properties of the fermionic reservoir are described by a single quantity, namely the spectral density $J_{R}(\omega)$, which can be generated by a superposition of weighted $\delta$ functions

$$
J_{R}(\omega)=\sum_{q} \pi\left|V_{q}\right|^{2} \delta\left(\omega-\omega_{q}\right) .
$$

Equation (18) becomes a smooth function for a dense spectrum of the reservoir modes. Using the properties of the $\delta$ function to transform the summation over $q$ into an integral over $\omega$ and extending its lower limit to $-\infty$ by assuming $J_{R}(\omega)=0$ for $\omega<0$, since we need an improper form of the integrals to solve them by applying the theorem of residues, we get the final integral equations for the reservoir correlation functions

$$
\begin{gathered}
C_{12}(t)=\int_{-\infty}^{\infty} \frac{\mathrm{d} \omega}{\pi} J_{R}(w) n_{F}\left(-\omega_{q}+E_{F}\right) e^{-i \omega t} . \\
C_{21}(t)=\int_{-\infty}^{\infty} \frac{\mathrm{d} \omega}{\pi} J_{R}(w) n_{F}\left(\omega_{q}-E_{F}\right) e^{i \omega t}
\end{gathered}
$$

To solve these integrals, we will pursue a method proposed by Meier and Tannor ${ }^{35}$ for bosonic systems and extend it to fermionic systems using a numerical decomposition of the spectral density for $\omega \geq 0$

$$
J_{R}(\omega)=\sum_{k=1}^{m} \frac{p_{k}}{4 \Omega_{k}} \frac{1}{\left(\omega-\Omega_{k}\right)^{2}+\Gamma_{k}^{2}},
$$


with real fitting parameters $p_{k}, \Omega_{k}$ and $\Gamma_{k}$. This decomposition is not restricted to a certain shape of the spectral density and can therefore be used to approximate complicated band structures. This enables one to avoid the assumption of the wide-band limit and to take influences of the band structure on the dissipative electron transfer between the wire and the leads fully into account.

With the complex roots of the Fermi function and of function (21), the theorem of residues applied to Eqs. (19. 20) results in

$$
\begin{aligned}
C_{12}(t)= & \sum_{k=1}^{m} \frac{p_{k}}{4 \Omega_{k} \Gamma_{k}}\left(n_{F}\left(-\Omega_{k}^{-}+E_{F}\right) e^{-i \Omega_{k}^{-} t}\right) \\
- & \frac{2 i}{\beta} \sum_{k}^{m^{\prime}} J_{R}\left(\nu_{k}\right) e^{-i \nu_{k} t} \\
C_{21}(t)= & \sum_{k=1}^{m} \frac{p_{k}}{4 \Omega_{k} \Gamma_{k}}\left(n_{F}\left(\Omega_{k}^{+}-E_{F}\right) e^{i \Omega_{k}^{+} t}\right) \\
& -\frac{2 i}{\beta} \sum_{k}^{m^{\prime}} J_{R}\left(\nu_{k}\right) e^{i \nu_{k} t}
\end{aligned}
$$

with the abbreviations $\Omega_{k}^{+}=\Omega_{k}+i \Gamma_{k}$ and $\Omega_{k}^{-}=\Omega_{k}-i \Gamma_{k}$ and the Matsubara frequencies $\nu_{k}$ given by $\nu_{k}=i \frac{2 \pi k+\pi}{\beta}+$ $E_{F}$. These equations determine the coefficients necessary to finally write the correlation functions as a superpostion of weighted exponential functions:

$$
\begin{aligned}
& C_{12}(t)=\sum_{k=1}^{m+m^{\prime}} a_{12}^{k} e^{\gamma_{12}^{k} t} \\
& C_{21}(t)=\sum_{k=1}^{m+m^{\prime}} a_{21}^{k} e^{\gamma_{21}^{k} t} .
\end{aligned}
$$

Rigorously, the sum over the Matsubara frequencies would be infinite but it can be truncated at a finite value depending on the temperature of the system $T$ and the spectral width of $J_{R}(\omega)$. This representation for the correlation function allows us to derive a set of differential equations for the auxiliary density operators (13, 14), viz.

$$
\begin{aligned}
\frac{\partial}{\partial t} \Lambda_{x x^{\prime}}^{k}(t)= & a_{x x^{\prime}}^{k} K_{x^{\prime}} \rho_{S}(t)-i\left[H_{S}(t), \Lambda_{x x^{\prime}}^{k}(t)\right] \\
& +\gamma_{x x^{\prime}}^{k} \Lambda_{x x^{\prime}}^{k}(t), \\
\frac{\partial}{\partial t} \widehat{\Lambda}_{x x^{\prime}}^{k}(t)= & \left(a_{x^{\prime} x}^{k}\right)^{*} \rho_{S}(t) K_{x^{\prime}}-i\left[H_{S}(t), \widehat{\Lambda}_{x x^{\prime}}^{k}(t)\right] \\
& +\left(\gamma_{x^{\prime} x}^{k}\right)^{*} \widehat{\Lambda}_{x x^{\prime}}^{k}(t)
\end{aligned}
$$

with $\Lambda_{x x^{\prime}}(t)=\sum_{k=1}^{m+m^{\prime}} \Lambda_{x x^{\prime}}^{k}(t)$ and $\widehat{\Lambda}_{x x^{\prime}}(t)=$ $\sum_{k=1}^{m+m^{\prime}} \widehat{\Lambda}_{x x^{\prime}}^{k}(t)$. These equations can be solved numerically using a simple Runge-Kutta method without the need of diagonalizing the Hamiltonian and together with Eq. (15), one now has a complete set of differential equations describing the population dynamics in the wire coupled to the lead in second-order perturbation theory for an arbitrary time-dependent wire Hamiltonian. Regarding the right lead, one just needs to add a second dissipation term to the master equation (15) with differently defined $K_{x}$ operators, i.e. acting on the last wire site $N$, and a second corresponding set of differential equations for the auxiliary operators.

\section{CURRENT EQUATION}

An intuitive approach to the electric current equation is to consider the rate of change of the number of electrons, with elementary charge $e$, inside the lead which reads in the density matrix formalism $17,18,19,20,24,26,27,49,50,51,52,53$

$$
I_{l}(t)=e \frac{\mathrm{d}}{\mathrm{d} t} \operatorname{tr}\left\{N_{l} \rho(t)\right\}=-i e \operatorname{tr}\left\{\left[N_{l}, H(t)\right] \rho(t)\right\}
$$

Here $N_{l}=\sum_{q} c_{q}^{\dagger} c_{q}$ denotes the electron number operator of the left lead with the summation performed over the reservoir degrees of freedom. Similar to the last sections, all calculations refer only to the left lead but are also valid for the right lead by adding the corresponding terms to the final set of differential equations. The trace and the density operator $\rho$ in Eq. (28) are defined in the Hilbert space of the full system consisting of wire and lead. Solving the commutator in Eq. (28) and making use of the equilibrium condition of the fermionic reservoir results in $I_{l}(t)=-2 e \sum_{q} \operatorname{Im} \operatorname{tr}\left\{V_{q} c_{1}^{\dagger} c_{q} \rho(t)\right\}$. Since the trace is invariant under a transformation into the interaction picture, $\tilde{A}(t)=U_{S}\left(t, t_{0}\right) A$ for any operator $A$ defined in the Hilbert space of the full system, one can use the integrated form of the Liouville equation in the interaction picture $\tilde{\rho}(t)=\tilde{\rho}\left(t_{0}\right)-i \int_{t_{0}}^{t} \mathrm{~d} t^{\prime}\left[\tilde{H}_{S R}\left(t^{\prime}\right), \tilde{\rho}\left(t^{\prime}\right)\right]$ for the time evolution of the full density operator $\tilde{\rho}(t)$ in the interaction representation. After solving all the commutator relations, separating the system part from the reservoir part and employing again the definition of the correlation functions (12), the ansatz for the current (28) finally reads

$$
\begin{array}{r}
I_{l}(t)=2 e \operatorname{Re} \int_{t_{0}}^{t}{ }^{t} t^{\prime}\left(\operatorname{tr}_{\mathrm{S}}\left\{\tilde{c}_{1}^{\dagger}(t) \tilde{c}_{1}\left(t^{\prime}\right) \tilde{\rho}_{S}\left(t^{\prime}\right)\right\} C_{12}\left(t-t^{\prime}\right)\right. \\
\left.-\operatorname{tr}_{\mathrm{S}}\left\{\tilde{c}_{1}\left(t^{\prime}\right) \tilde{c}_{1}^{\dagger}(t) \tilde{\rho}_{S}\left(t^{\prime}\right)\right\} C_{21}^{*}\left(t-t^{\prime}\right)\right) .
\end{array}
$$

The current information is partly contained in the temporal phase relation between the annihilation and creation operators which can be incorporated into a single time evolution operator $U_{S}$, defined in the Hilbert space of the relavant system, by using the cyclic properties of the 
trace to write Eq. (29) as

$$
\begin{array}{r}
I_{l}(t)=2 e \operatorname{Re}\left(\operatorname{tr}_{S}\left\{c_{1}^{\dagger} \int_{t_{0}}^{t} \mathrm{~d} t^{\prime} U_{S}^{\dagger}\left(t^{\prime}, t\right) c_{1} \rho_{S}\left(t^{\prime}\right)\right\} C_{12}\left(t-t^{\prime}\right)\right. \\
\left.-\operatorname{tr}_{S}\left\{c_{1}^{\dagger} \int_{t_{0}}^{t} \mathrm{~d} t^{\prime} U_{S}^{\dagger}\left(t^{\prime}, t\right) \rho_{S}\left(t^{\prime}\right) c_{1}\right\} C_{21}^{*}\left(t-t^{\prime}\right)\right) \cdot(
\end{array}
$$

These integrals have the same structure as the auxiliary density matrices defined in the last section and can be expressed in terms of $\Lambda_{12}(t)$ and $\widehat{\Lambda}_{12}(t)$. Thus, we get the final equation for the time-dependent current

$$
I_{l}(t)=2 e \operatorname{Re}\left(\operatorname{tr}_{S}\left\{c_{1}^{\dagger} \Lambda_{12}(t)-c_{1}^{\dagger} \widehat{\Lambda}_{12}(t)\right\}\right)
$$

between the left lead and the first site of the wire. Similar to the master equation (15) for the population dynamics, all the information about the interaction of the system with the reservoir is contained in the time-dependent auxiliary matrices (13, 14) weighting the corresponding system operator. Eq. (31) is valid within the framework of assumptions we have made to derive Eq. (15) and allows one to measure the time-resolved current for arbitrary time-dependent systems.

The EOM for the required auxiliary operators are already known from the time propagation of the population. Thus, we have a complete set of differential equations consisting of Eqs. (26] 27), the master equation (15) and the current equation (31) and therefore an applicable formalism to compute the current and the population dynamics for a wide range of systems. The same set of equations has to be applied to the last site of the wire as well to describe a wire enclosed by two leads, as shown in Fig. 1 Furthermore, the ability to define any desired number of leads (with respect to the given computational resources) makes it possible to realize driven current switches with an arbitrarily complicated configuration.

\section{ALTERNATIVE TIME-LOCAL APPROACH}

Since we started with the Nakajima-Zwanzig identity to derive our equations for the population dynamics the final expressions are in a TNL form. In this section, we shortly present their TL counterparts without giving an extended comparison of both approaches. In the literature, the TL approach is also known as time-convolutionless formalism ${ }^{45}$, partial time ordering prescription ${ }^{42,43.44}$ or Tokuyama-Mori approach ${ }^{54}$. Using the Tokuyama-Mori identity one gets in second-order perturbation theory for the wire-lead coupling the following equations for the population dynamics of the wire,

$$
\begin{aligned}
\dot{\rho}_{S}(t)= & -i \mathcal{L}_{S}(t) \rho_{S}(t) \\
& -\sum_{x x^{\prime}}\left[K_{x}, \Lambda_{x x^{\prime}}(t) \rho_{S}(t)-\rho_{S}(t) \widehat{\Lambda}_{x x^{\prime}}(t)\right]
\end{aligned}
$$

with the modified corresponding auxiliary operators

$$
\begin{aligned}
& \Lambda_{x x^{\prime}}(t)=\int_{t_{0}}^{t} \mathrm{~d} t^{\prime} C_{x x^{\prime}}\left(t-t^{\prime}\right) U_{S}\left(t, t^{\prime}\right) K_{x^{\prime}} \\
& \widehat{\Lambda}_{x x^{\prime}}(t)=\int_{t_{0}}^{t} \mathrm{~d} t^{\prime} C_{x^{\prime} x}^{*}\left(t-t^{\prime}\right) U_{S}\left(t, t^{\prime}\right) K_{x^{\prime}} .
\end{aligned}
$$

One can also derive these equations by just applying the substitution $\rho\left(t^{\prime}\right)=U_{S}^{\dagger}\left(t, t^{\prime}\right) \rho(t)$ to the corresponding set of TNL equations ${ }^{39}$, neglecting the influence of dissipation during the time propagation of the density operator within the integral. Thus, the current equation reads $\frac{19,20}{}$

$$
I_{l}(t)=2 e \operatorname{Re}\left(\operatorname{tr}_{S}\left\{c_{1}^{\dagger} \Lambda_{12}(t) \rho_{S}(t)-c_{1}^{\dagger} \rho_{S}(t) \widehat{\Lambda}_{12}(t)\right\}\right) .
$$

TNL and TL approaches for similar systems have been compared in recent numerical studies ${ }^{39.55}$ and it depends strongly on the coupling parameters which of both is favorable. Within the parameter regime we used for the following numerical investigations, TL and TNL calculations showed almost identical results.

\section{NUMERICAL RESULTS FOR THE LASER DRIVEN MOLECULAR WIRE}

The system depicted in Fig. 1 1 represents a simple configuration which allows one to study a variety of transport phenomena. The following numerical results are expressed in terms of the tight-binding hopping parameter $\Delta$, where $\Delta=0.1 \mathrm{eV}$ is a reasonable value for molecular systems 17.24 .26 .27 .50 .51 .52 .53 . The system temperature is set to $T=0.25 \Delta / k_{B}=290 \mathrm{~K}$. Despite the fact that it would be possible in Eq. (21), we do not simulate a realistic spectrum for the coupling between the leads and the wire, but rather restrict the spectrum to a single Lorentzian. For example, a realistic gold s-band would be a superposition of different Lorentzians forming certain band edges. Taking only one Lorentzian into account corresponds to a rough approximation of only one band edge, but this makes the system and the underlying physical processes easier to understand. Due to the weak coupling requirement, the spectral density should be about one order smaller than the internal dynamics, thus the peak of the Lorentzian $J_{R}(\omega)$ is set to $0.1 \Delta$, what is guaranteed by the condition $p_{1}=0.1\left(4 \Delta \Omega_{1} \Gamma_{1}^{2}\right)$. Reasonable values for the bandwith parameters $\Gamma_{k}$ are in the region of $10 \mathrm{eV}$. With the chosen energy settings, a time unit in the system corresponds to $0.66 \mathrm{fs}$ what enables one to resolve time-dependent effects on a femtosecond scale. The resulting current unit can be extracted from Eq. (31) and is equal to a macroscopic value of $1[e]=2.43 * 10^{-4} \mathrm{~A}$.

As a basis set for the tight-binding Hamiltonian we choose the many-body Fock space in which each site is represented by a two-state vector $\left|\chi_{n}\right\rangle$. The set of these 
state vectors forms the total state vector $|\Psi\rangle$ by the tensor product $|\Psi\rangle=\left|\chi_{1}\right\rangle \otimes\left|\chi_{2}\right\rangle \otimes \ldots \otimes\left|\chi_{N}\right\rangle$. The corresponding annihilation and creation operators for site $n$

$$
c_{n}^{\dagger}=\left(\begin{array}{ll}
0 & 0 \\
1 & 0
\end{array}\right), \quad c_{n}=\left(\begin{array}{ll}
0 & 1 \\
0 & 0
\end{array}\right)
$$

represent a complete operator basis for the master and the current equation.

\section{A. The transient oscillation of the undriven wire}

The equilibrium condition of the on-site population of the wire in the absence of a second lead is determined by the Fermi function $n_{F}$ taken at the on-site energy of the site, e.g. $\operatorname{tr}\left(c_{1}^{\dagger} c_{1} \rho_{S}(t)\right)=n_{F}\left(E_{1}-E_{F}\right)$. In this particular case, the current drops to zero after the wire is filled to its equilibrium value, simply due to the lack of a closed electrical circuit. The situation is more interesting in the case where two leads are coupled to the wire, as it is shown in Fig. 1 Fig. 2] shows a situation for a wire with two sites, in which the wire states at time zero are unoccupied. The equilibrium state is reached after a relaxation time that mostly depends on the wire-lead coupling strength. While the number of electrons in the relevant system is not constant in time, the trace of the reduced density operator is conserved and normalized. A bias voltage on the system can be simulated by setting a difference between the left and right Fermi energy, here $E_{F, l}-E_{F, r}=10 \Delta$. Since we set the on-site energies to $E_{1}=E_{2}$ and apply a weak coupling scheme where the internal dynamics of the inner system is about one order faster than the electron transfer between the leads and the wire, a spatial drop of the population within the wire in its equilibrium state cannot be observed in the upper panel of Fig. 2 The equilibrium population of the entire wire is determined by the average over the expectation values of the left and right sites, which is given by $\operatorname{tr}\left(c_{1}^{\dagger} c_{1} \rho_{S}(t)\right)=$ $\operatorname{tr}\left(c_{2}^{\dagger} c_{2} \rho_{S}(t)\right)=\left(n_{F}\left(E_{1}-E_{F, l}\right)+n_{F}\left(E_{2}-E_{F, r}\right)\right) / 2$ if the spectral densities of both leads, i.e. their coupling to the wire, are the same. The transient oscillations of the corresponding directed net current decay and a constant equilibrium value is reached, when the relaxation process is over. The upper bound for the net current is determined by the small wire-lead coupling strength, namely the spectral function $J_{R}(\omega)$ with its maximum value of $0.1 \Delta$.

\section{B. AC voltage}

Time-dependent electronic reservoirs in molecular systems can be modelled within the Floquet theory by applying a gauge transformation on the wire Hamiltonian ${ }^{56}$ or by using a source-Redfield ${ }^{18}$ equation. Despite the fact that we initially assumed that the lead Hamiltonian

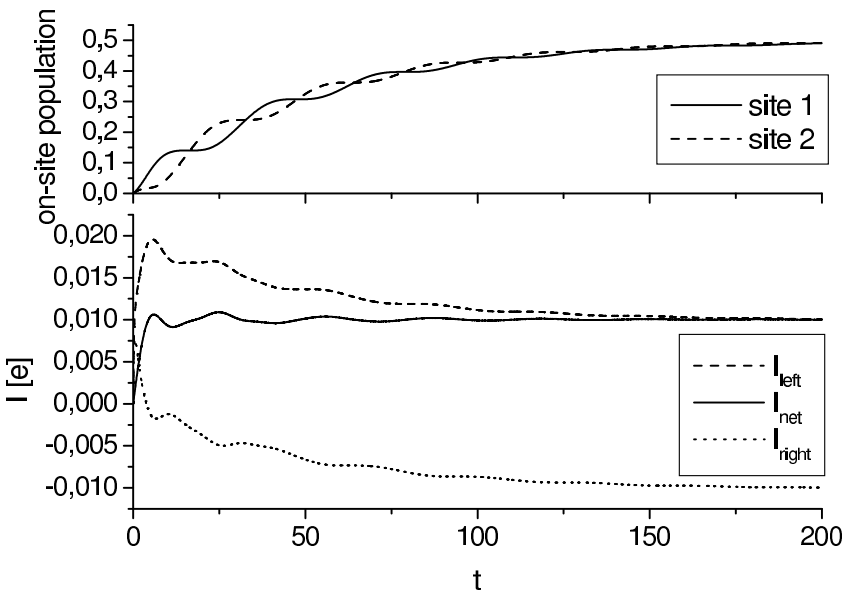

FIG. 2: Upper panel: population dynamics measured by $\operatorname{tr}\left(c_{n}^{\dagger} c_{n} \rho_{S}(t)\right)(n=1,2)$ for the system with two sites without driving field $U(t)$ as a function of time $t$ starting with unoccupied wire states. The applied DC voltage is realized by a difference between the left and the right Fermi energy of $E_{F, l}-E_{F, r}=10 \Delta$. The on-site energies of the wire are aligned and centered between the left and the right Fermi energy $E_{1}=E_{2}=E_{F, r}+5 \Delta=E_{F, l}-5 \Delta$. Lower panel: the corresponding currents flowing from the left lead into the first site $I_{\text {left }}$, from the right lead into right site $I_{\text {right }}$ and the net current given by $I_{\text {net }}=\left(I_{\text {left }}-I_{\text {right }}\right) / 2$.

is time-independent, the formalism allows one to treat time-dependent lead Hamiltonians as well by simply considering time-dependent coefficients for the correlation functions $C(t)=\sum_{k} a_{k}(t) e^{\gamma_{k}(t) t}$, in the case of the TL theory, and a time derivative given by

$$
\begin{aligned}
\frac{\mathrm{d}}{\mathrm{d} t} C(t)= & \sum_{k}^{m+m^{\prime}} a_{k}(t) e^{\gamma_{k}(t) t}\left(\gamma_{k}(t)+\dot{\gamma}_{k}(t) t\right) \\
& +\sum_{k}^{m+m^{\prime}} \dot{a}_{k}(t) e^{\gamma_{k}(t) t}
\end{aligned}
$$

To get the required form $\dot{C}(t)=\gamma_{k}(t) C(t)$ which is necessary to derive differential equations for Eqs. (13) 14), we have to assume that

$$
\begin{gathered}
\left|\dot{a}_{k}(t)\right| \ll\left|a_{k}(t) \gamma_{k}(t)\right|, \\
\left|e^{\gamma_{k}(t) t} \dot{\gamma}_{k}(t) t\right| \ll\left|e^{\gamma_{k}(t) t} \gamma_{k}(t)\right|,
\end{gathered}
$$

for all times $t$ not negligible in the time integration of the auxiliary operators in Eqs. 13 14. In general, this assumption corresponds to the statement that the lead dynamics is sufficiently slower than the lead-wire coupling dynamics. The lead-wire coupling can be influenced by a variation of the chemical potential of the electron distribution in the leads, by changing the parameters $\Omega_{k}, \Gamma_{k}, p_{k}$ of the spectral decomposition, or both. A change in the chemical potential and keeping the spectral 


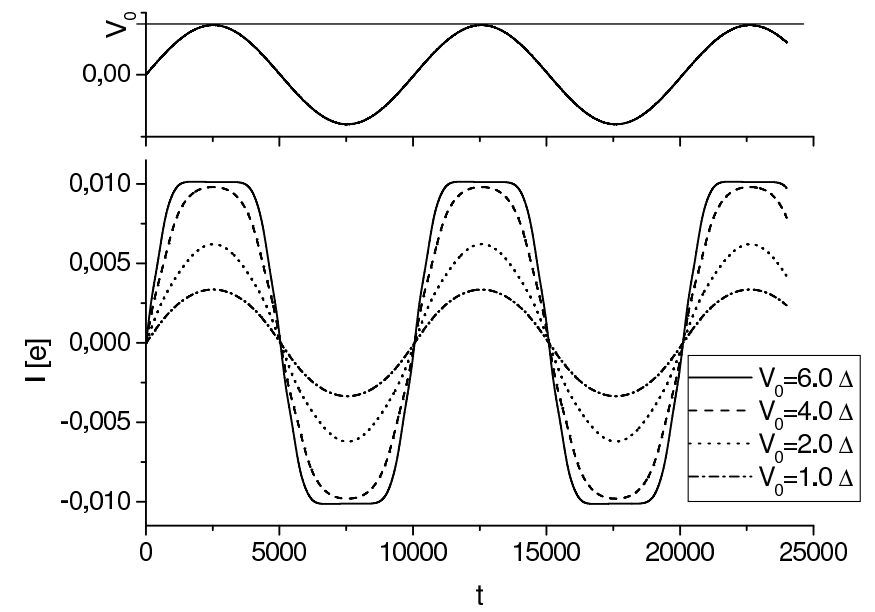

FIG. 3: The AC voltage (upper panel) with amplitude $V_{0}$ and frequency $\omega_{V}=0.00625 \Delta$ drives an AC $I[e]$ (bottom panel) between the left and the right lead through an undriven and not disordered wire consisting of five sites. A current cut-off appears for large AC voltages, since the wire-lead coupling is weak.

function unchanged would correspond to a charging process, while shifting the spectral density, i.e. increasing or decreasing the $\Omega_{k}$ parameters, and the chemical potential by the same value describes a total change of the electrostatic potential without an alteration of the population in the leads. In the present work, we restrict ourselves to a time-periodic modulation of the difference between the chemical potentials of the left and right lead $E_{F, r}-E_{F, l}=$ $V_{0} \sin \left(\omega_{V} t\right)$, thereby approximating an AC voltage on the leads with amplitude $V_{0}$. In this special case, the quality of the approximation of slow dynamics can be determined considering the general time dependence of the coefficients given by Eqs. (22] 23) as $\left|a_{k}(t)\right| \sim\left|n_{F}\left(E_{F}(t)\right)\right|$ for the non-Matsubara terms with the corresponding time-independent exponents $\gamma_{k}$. Therefore, the time derivative becomes $\left|\dot{a}_{k}(t)\right| \sim\left|n_{F}^{2}\left(E_{F}(t)\right) \beta \dot{E}_{F}(t)\right| \sim$ $\left|\frac{V_{0} \omega_{V}}{T} \cos \left(\omega_{V} t\right) n_{F}^{2}\left(E_{F}(t)\right)\right|$ and conditions (38, 39) are justified if $\left|\frac{V_{0} \omega_{V}}{T}\right| \ll 1$. Regarding the Matsubara terms, the relevant time-dependent coefficients are $\gamma_{k}(t)=$ $-(2 \pi k+\pi) T+i E_{F}(t)$ and $\left|a_{k}(t)\right|=\left|2 T J\left(\nu_{k}\right)\right|$ for $k=$ $1 \ldots m^{\prime}$ and it can be shown that conditions (38) 39) are also fulfilled if $\frac{V_{0} \omega_{V}}{(\pi+2 \pi k) T} \ll 1$ which complies with the condition for the non-Matsubara coefficients. A disregard of the conditions (38) 39) would cause additional fast oscillations of the current during those times in which the $\mathrm{AC}$ voltage changes rapidly. The $\mathrm{AC}$ voltage causes an $\mathrm{AC}$ in the system, both shown in Fig. [3 that follows the driving voltage. For large voltage amplitudes $V_{0}$ a finite cut-off appears due to a bottle neck of the system given by its weak coupling to the electronic leads.

\section{Coherent destruction of tunneling}

Coherent destruction of tunneling $24,26.52 .53 .57$ (CDT) is a well understood quantum mechanical effect where an external periodic driving field of the form

$$
A(t)=A_{0} \sin \left(\omega_{d} t\right)
$$

yielding an asymmetric potential $U_{n}(t)=A(t) \delta_{1 n}-$ $A(t) \delta_{2 n}$ in Eq. (3) can suppress the time-averaged current in the driven two-site system depicted in Fig. 1] The current completely breaks down if the ratio of the field parameters $A_{0} / \hbar \omega_{d}$ is equal to a zero of the Bessel function $J_{0}$ (e.g. $2.405,5.520,8.654, \ldots$ ), a condition that holds for isolated quantum systems as well as for open systems 24.26 .52 .53 . This is a simple but nontrivial system for a time-dependent conduction formalism and we extend the results of former calculations which applied the wide-band approximation ${ }^{24,26.52,53}$ to finite band effects by using a spectral decomposition. The finite width of the decomposed spectral density (21) causes an additional decay of the current with increasing amplitude since the coupling strength between the lead and the corresponding site decreases when the on-site energy of the coupled site is driven to the edges of the Lorentzian during the oscillation generated by the external field $A(t)$. Naturally, this effect becomes more dominant for a smaller bandwidth parameter $\Gamma_{1}$, shown in Fig. 4 The time-averaged current shows the predicted breakdowns at the zeros of $J_{0}$. The amplitude $A_{0}$ of the applied electrical field is measured in dimensions of $[\Delta]$ which corresponds to field strengths of about $10^{8} \frac{\mathrm{V}}{\mathrm{cm}}$ by assuming intra-atomar distances of $1 \AA$. The driving frequency is set to $\omega_{d}=10 \Delta$ which is in the low energy branch of infrared light. Here the bias voltage is set to $E_{F, l}-E_{F, r}=60 \Delta=6 \mathrm{eV}$.

\section{Optical current switching}

The major advantage of the derived conduction formalism, in addition to the avoidance of the wide-band limit, is the applicability to unrestricted time-dependent systems. Former CDT studies of currents in open quantum systems ${ }^{24.26 .53}$ were based on an infinite-time averaging of the currents due to the mathematical nature of the used approaches. In Fig. 5 we apply a finite laser pulse with a Gaussian shaped amplitude

$$
A(t)=A_{0} \exp \left(\frac{-(t-T)^{2}}{2 \sigma^{2}}\right) \sin \left(\omega_{d} t\right)
$$

to the asymmetric driven system described in the last subsection. The peak amplitude of the Gaussian was set to $A_{0}=24.05 \Delta$, where the CDT relation applies. This finite laser pulse causes the time-resolved current to oscillate around zero, shown in the center panel of Fig. [5] The situation becomes more obvious by looking at the time averaged current depicted in the bottom panel of 


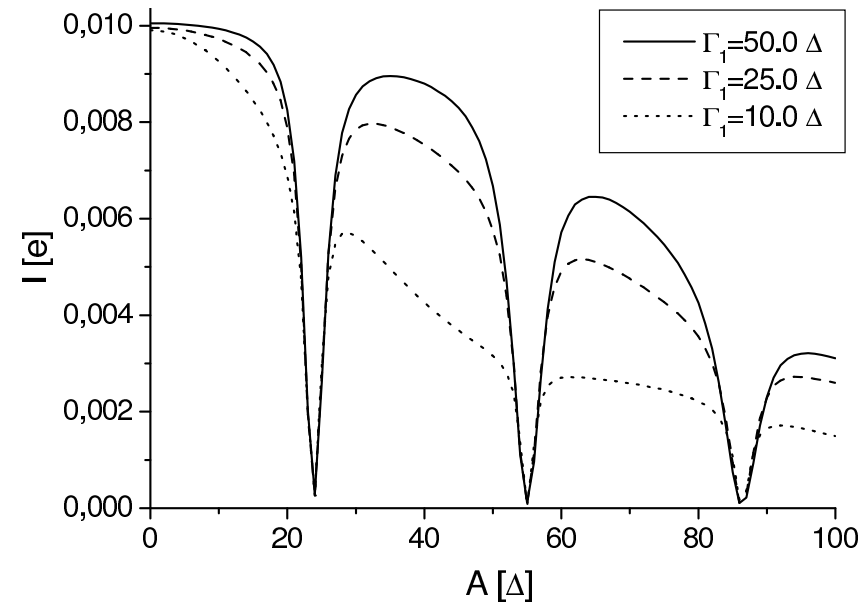

FIG. 4: The time-averaged current for the periodically driven two-site system as a function of the amplitude for different bandwith parameters $\Gamma_{1}$. The frequency of the external field is $\omega_{d}=10 \Delta$. The applied bias voltage is $60 \Delta$. The Fermi energies $E_{F, r}=\Omega_{1}-30 \Delta, E_{F, l}=\Omega_{1}+30 \Delta$ and the on-site energies $E_{1}=E_{2}=\Omega_{1}$ are set in relation to the parameter $\Omega_{1}$ of the spectral density.

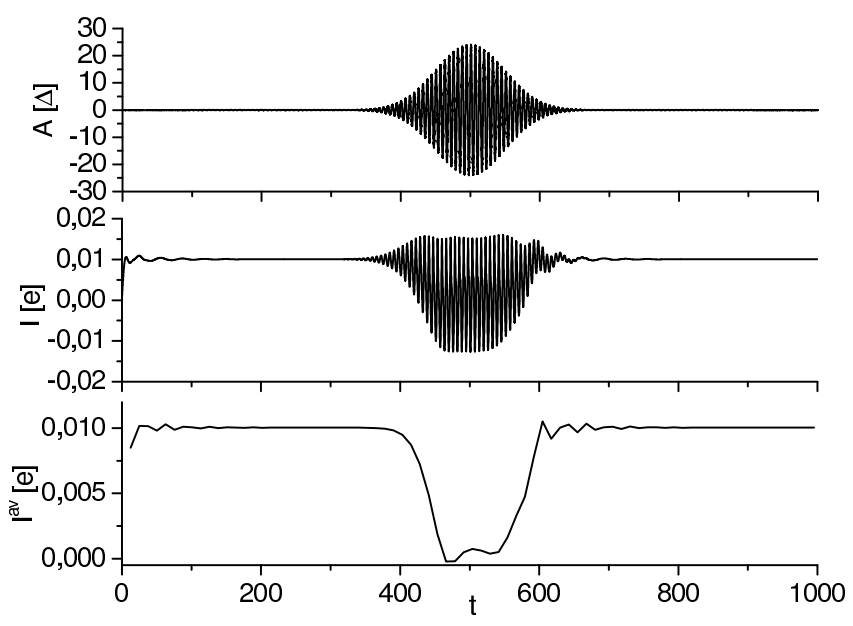

FIG. 5: The upper panel shows the Gaussian excitation pulse with a peak amplitude of $A_{0}=24.05 \Delta$ and a width of $\sigma^{2}=$ 50. It excites the time-resolved net current depicted in the middle panel. If the net current is averaged over three periods of the fast pulse oscillation, a complete suppression can be observed, depicted in the bottom panel. The on-site energies are equal $E_{1}=E_{2}=\Omega_{1}$ and centered between the left and the right Fermi energy, which define a DC voltage of $E_{F, r}-E_{F, l}=$ $10 \Delta$.

Fig. 5] where the averaging was taken over three oscillations of the driving field. The averaged current is almost suppressed during the time in which the Gaussian laser field is applied to the system and CDT is performed with a finite laser pulse. The effect is superimposed by transient oscillations because the system is under the constraint of permanently changing variables and tries to find its equilibrium state. This finite-time effect gives rise to interesting experimental realizations and further investigations will deal with the idea to apply the current formalism to non-local optimal control algorithms to compute more sophisticated and applicable external control fields.

\section{SUMMARY AND CONCLUSION}

We have developed a time-dependent non-Markovian conduction formalism based on a projection operator approach for the density matrix with a numerical decomposition of the spectral density which allows one to study time-resolved currents and population dynamics in molecular wires for arbitrary time-dependent wire Hamiltonians, which is an useful extension to existing theories and applicable for a wide range of systems. The formalism includes the coupling to the electronic leads in second-order perturbation theory and a non-perturbative interaction with external fields. Its validity was shown for the example of coherent destruction of tunneling in a driven two-state system and further effects like optical control of current by using a short laser pulse, alternating currents and electrical relaxation of the system into a biased equilibrium state were presented. These effects were investigated on a femtosecond time scale, which is an important aspect for the the applicability of the formalism for future investigations of femtosecond spectroscopy, optical control and optimization of electrical currents. One can easily include electron-vibrational couplings in second-order perturbation theory and electronelectron correlations resulting in a powerful tool to simulate currents under quite realistic conditions as long as the weak coupling scheme is justified. Furthermore, recent publications ${ }^{19.20}$ suggest a way to avoid this weak coupling condition within the framework of the presented formalism.

\section{Acknowledgments}

We are grateful to Markus Schröder and Sigmund Kohler for useful discussions.
1 R. A. Marcus, J. Chem. Phys. 24, 966 (1956).

${ }^{2}$ W. T. Pollard, A. K. Felts, and R. A. Friesner, Adv. Chem. Phys. 93, 77 (1996).
3 M. Bixon and J. Jortner, Adv. Chem. Phys. 106\&107 (1999), special issue on electron transfer.

4 T. Mančal, U. Kleinekathöfer, and V. May, J. Chem. Phys. 
117, $636(2002)$.

5 A. Nitzan and M. A. Ratner, Science 300, 1384 (2003).

6 A. W. Ghosh, P. S. Damle, S. Datta, and A. Nitzan, MRS Bulletin 6, 391 (2004).

7 M. A. Reed, C. Zhou, C. J. Muller, T. P. Burgin, and J. M. Tour, Science 278, 252 (1997).

8 R. H. M. Smit, Y. Noat, C. Untiedt, N. D. Lang, M. C. Hemert, and J. M. van Ruitenbeek, Nature 419, 906 (2002).

9 J. Reichert, R. Ochs, H. B. Weber, M. Mayor, and H. von Löhneysen, Phys. Rev. Lett. 88, 176804 (2002).

10 V. Mujica, M. Kemp, and M. A. Ratner, J. Chem. Phys. 101, 6849 (1994).

11 A. Nitzan, Ann. Rev. Phys. Chem. 52, 681 (2001).

12 A. Nitzan, J. Phys. Chem. A 105, 2677 (2001).

${ }^{13}$ R. Landauer, IBM J. Res. Develop. 1, 233 (1957).

14 M. Büttiker, Phys. Rev. Lett. 57, 1761 (1986).

15 S. Datta and M. P. Anantram, Phys. Rev. B 45, 13761 (1992).

16 N. Wingreen, A. Jauho, and Y. Meir, Phys. Rev. B 48, 8487 (1993).

17 S. Kohler, J. Lehmann, and P. Hänggi, Phys. Rep. 406, 379 (2004).

18 I. V. Ovchinnikov and D. Neuhauser, J. Chem. Phys. 122, 024707 (2005).

19 X.-Q. Li, J.-Y. Luo, Y.-G. Yang, P. Cui, and Y. J. Yan, Phys. Rev. B 71, 205304 (2005).

${ }^{20}$ P. Cui, X.-Q. Li, J. Shao, and Y. J. Yan, cond-mat/0506477v1.

21 R. Gutiérrez-Laliga, S. Mandal, and C. Cuniberti, Phys. Rev. B 71, 235116 (2005).

${ }^{22}$ M. Č́žzek, M. Thoss, and W. Domcke, Phys. Rev. B 70, 125406 (2004).

23 M. Č́žzek, M. Thoss, and W. Domcke, Czech. J. Phys. 55, 189 (2005).

24 J. Lehmann, S. Kohler, V. May, and P. Hänggi, J. Chem. Phys. 121, 2278 (2004).

25 M. Galperin and A. Nitzan, cond-mat/0503114v2.

26 J. Lehmann, S. Camalet, S. Kohler, and P. Hänggi, Chem. Phys. Lett. 368, 282 (2003).

27 J. Lehmann, S. Kohler, P. Hänggi, and A. Nitzan, J. Chem. Phys. 118, 3283 (2003).

28 S. Kurth, G. Stefanucci, C.-O. Almbladh, A. Rubio, and E. K. U. Gross, Phys. Rev. B 72, 035308 (2005).

29 J. Dachnovsky, J. Chem. Phys. 100, 6492 (1994).

30 E. Geva, R. Kosloff, and J. L. Skinner, J. Chem. Phys. 102, 8541 (1995).

31 I. A. Goychuk, E. G. Petrov, and V. May, Chem. Phys.
Lett. 253, 428 (1996).

32 R. I. Cukier, C. Denk, and M. Morillo, Chem. Phys. 217, 179 (1997).

33 M. Grifoni and P. Hänggi, Phys. Rep. 304, 229 (1998).

34 D. Schirrmeister and V. May, Chem. Phys. Lett. 297, 383 (1998).

35 C. Meier and D. J. Tannor, J. Chem. Phys. 111, 3365 (1999).

36 S. Nakajima, Prog. Theor. Phys. 20, 948 (1958).

37 R. Zwanzig, in Lectures in Theoretical Physics, edited by W. E. Brittin, B. W. Downs, and J. Downs (Interscience, New York, 1961), vol. 3, p. 106.

38 R. Xu and Y. J. Yan, J. Chem. Phys. 116, 9196 (2002).

39 U. Kleinekathöfer, J. Chem. Phys. 121, 2505 (2004).

40 M. V. Korolkov and G. K. Paramonov, Phys. Rev. A 55, 589 (1997).

41 R. Zwanzig, Physica 30, 1109 (1964).

42 S. Mukamel, I. Oppenheim, and J. Ross, Phys. Rev. A 17, 1988 (1978).

43 D. R. Reichman, F. L. H. Brown, and P. Neu, Phys. Rev. E 55, 2328 (1997).

44 Y. J. Yan, Phys. Rev. A 58, 2721 (1998).

45 H. P. Breuer, B. Kappler, and F. Petruccione, Phys. Rev. A 59, 1633 (1999).

46 F. Haake, Springer Tracts Mod. Phys. 66, 98 (1973).

47 K. Blum, Density Matrix Theory and Applications (Plenum Press, New York, 1996), 2nd ed.

48 V. May and O. Kühn, Charge and Energy Transfer in Molecular Systems (Wiley-VCH, Berlin, 2000).

49 C. Bruder and H. Schoeller, Phys. Rev. Lett. 72, 1076 (1993).

50 J. Lehmann, S. Kohler, P. Hänggi, and A. Nitzan, Phys. Rev. Lett. 88, 228305 (2002).

51 J. Lehmann, G.-L. Ingold, and P. Hänggi, Chem. Phys. 281, 199 (2002).

52 S. Camalet, S. Kohler, and P. Hänggi, Phys. Rev. B 70, 155326 (2004).

53 S. Kohler, S. Camalet, M. Strass, J. Lehmann, G. L. Ingold, and P. Hänggi, Chem. Phys. 296, 243 (2004).

54 V. Cápek, Physica A 203, 520 (1994).

55 Y.-J. Yan and R.-X. Xu, Ann. Rev. Phys. Chem. 56, 187 (2005).

56 S. Kohler, J. Lehmann, and P. Hänggi, Phys. Rep. 406, 379 (2005).

57 F. Grossmann, T. Dittrich, P. Jung, and P. Hänggi, Phys. Rev. Lett. 67, 516 (1991). 